\title{
Role of Out-of-Plane Copper and Thallium Orbitals in Thallium Cuprate
}

\author{
F. Studer*, C. Gasser*, H. Murray*, P. Srivastava**, B.R. Sekhar***, K.B. Garg** and C.T. Chen*** \\ * CRISMAT-ISMRA, 14050 Caen cedex, France \\ ** CMPL, University of Rajasthan, Jaipur 300004, India \\ *** SRRC, Hsinchu Science Park, Hsinchu 30077, Taiwan
}

\begin{abstract}
Simultaneous total and fluorescence yield XAS measurements on $\mathrm{Cu} \mathrm{L}_{3}$-edge and, for the first time, total yield measurements at $\mathrm{Tl} \mathrm{L}_{3}$-edge of well oriented $\mathrm{Tl}(2212)$ thin films are reported in this work. Also, $\mathrm{Cu} \mathrm{L}_{3}$ and $\mathrm{O} \mathrm{K}$-edge measurements on overdoped $\mathrm{Tl}(2212)$ thin films using bulk sensitive fluorescence yield detection mode are reported showing that large out-of-plane hole densities do exist in the thallium cuprates at least for overdoped samples. Our results suggest that use of a bulk sensitive technique is necessary in order to get a reliable estimation of states having $\mathrm{Cu} 3 \mathrm{~d}_{\mathrm{z}}{ }^{2}-\mathrm{r}{ }^{2}$ character and thus a precise knowledge of sample stoichiometry. No direct correlation is found between $\mathrm{Tc}$ and out-of-plane covalent and doping hole densities such that theories based on the existence of large fraction of these out-of-plane unoccupied states are ruled out. To understand the role of out-of-plane copper orbitals, polarization dependent measurements of $\mathrm{Cu}_{\mathrm{L}}$-absorption edge on well characterised $\mathrm{Tl}(2212)$ thin films have been performed. The density of unoccupied states having $3 \mathrm{~d}_{z}{ }^{2}-\mathrm{r}^{2}$ character is found to depend on the amount of doping suggesting that out-of-plane copper orbitals essentially play a role of hole reservoir. The energy shift in the positions of white lines of $E / /(a, b)$ and $E / / c$ spectra is also found to be doping dependent. The probable reason for the observed shift is discussed.
\end{abstract}

\section{INTRODUCTION}

The $\mathrm{Bi}(2212)$ and $\mathrm{Tl}(2212)$ have notable differences in their electronic structure and superconducting properties inspite of having a similar crystal structure with three types of oxygen atoms located in $\mathrm{CuO} 2[\mathrm{O}(1)], \mathrm{Sr}(\mathrm{Ba}) \mathrm{O}[\mathrm{O}(2)]$ and $\mathrm{Bi}(\mathrm{Tl}) \mathrm{O}[\mathrm{O}(3)]$ layers. In $\mathrm{Tl}(2212)$, out-of-plane bond lengths of $\mathrm{Tl}-\mathrm{O}(2)$ and $\mathrm{Tl}-\mathrm{O}(3)$ are shorter than the in-plane bond lengths of $\mathrm{Tl}-\mathrm{O}(3)$ $[1,2]$. This makes charge transfer and interlayer coupling quite probable $[3,4]$. However, till now Tl-cuprates are not as extensively studied as the Bi-cuprates because of complexities involved in the preparation of high quality thallium thin films and single crystals. In our previous contribution [5], we have reported polarized $\mathrm{Cu} \mathrm{L}_{3}$ measurements on well oriented $\mathrm{Tl}(2212$ ) thin film in order to study symmetry and density of doping holes and their influence on Tc. The doping holes were found to have only in-plane symmetry. A previous comparison between TY and FY detection modes $\mathrm{Cu}_{3} \mathrm{~L}_{3}$-edges of $\mathrm{Tl}(2212)$ thin films has shown no differences in the $\mathrm{E} / /(\mathrm{a}, \mathrm{b})$ spectra (normal incidence) after full correction for the self reabsorption efeects in FY mode but significant difference in the $\mathrm{E} / / \mathrm{c}$-axis (grazing incidence) due to surface effects.

In the present contribution, using the FY detection mode polarized $\mathrm{Cu} \mathrm{L}_{3}$ measurements on three different $\mathrm{Tl}(2212)$ thin films synthesized and annealed in different conditions and $O \mathrm{~K}$-edge measurement on one thin film are presented. These measurements were performed at two different beamlines. Using these, the existence and role of doping hole density in the outof-plane orbitals is discussed in Tl bilayer cuprates.

\section{EXPERIMENTAL}

The thin films were deposited on $\mathrm{LaAlO}_{3}(100)$ oriented by multitarget sputtering. The details of the deposition process is described elsewhere [6]. The films were found to be monophasic by $x$-ray diffraction and exhibit homogeneous platelets with the c-axis perpendicular to the substrate plane. The magnetic susceptibility data indicate Tc of $105 \mathrm{~K}$ for the post annealed thin film and $101 \mathrm{~K}$ for the remaining two.

The $\mathrm{Cu} \mathrm{L}_{3}$-edge spectra have been recorded at room temperature both in the Total and Fluorescence Yield modes on the SA32 line of the SUPER ACO facility (LURE, Orsay) operating at $800 \mathrm{MeV}$ and $200 \mathrm{~mA}$. The XAS measurements for one of the thin films were performed at AT\&T Bell Labs, Dragon beamline at NSLS, Brookhaven National Lab. A standard procedure has been adopted to remove the background contribution, and the normalization point was chosen on the continuum at $948 \mathrm{eV}$.

\section{RESULTS AND DISCUSSION}

\subsection{Cu L3-EDGE}

\subsubsection{Existence of doping hole density in out-of-plane Cu orbitals}

$\mathrm{Cu} \mathrm{L}_{3}$ spectra measured on a high oxygen pressure $(70$ bars) annealed $\mathrm{T}(2212)$ thin film is shown in figure 1 . The spectra were recorded on the Dragon beamline at BNL. The appreciable amount of the $3 \mathrm{~d}^{9} \underline{\mathrm{L}}$ peak at $75^{\circ}$ is very much evident, clearly depicting the existence of out-of-plane doping holes. After full correction for the self-absorption in soft fluorescence extended $\mathrm{X}$-ray fine structure, the spectra were then simulated and using the dipolar angular variation form density of covalent and doping holes along the z-axis were estimated. The covalent and doping hole density along the z-axis are $25 \%$ and $15 \%$ respectively. This is quite an appreciable amount in comparison to the thin film synthesized under low oxygen pressure (Fig.2) which shows $10 \%$ and $0 \%$ covalent and doping holes along the z-axis. However the Tc increases only from $100 \mathrm{~K}$ to $105 \mathrm{~K}$ from the former to the latter. This implies that, after a certain amount of doping, exchange do take place between $\mathrm{Cu}$ and its apical oxygens, leading one to assume that $3 \mathrm{~d}_{\mathrm{z}}{ }^{2}-\mathrm{r}^{2}$ orbital mixes with $\mathrm{O} 2 \mathrm{p}$ valence band. The probable explanation of detection of 
doping holes having $3 \mathrm{~d}_{z^{2}-\mathrm{r}^{2}}$ symmetry in these overdoped $\mathrm{Tl}(2212)$ thin films can be derived from the fact that the extra oxygen holes change the environment around $\mathrm{Cu}$ resulting in the reduction of asymmetry of crystal field. This in turn reduces the splitting between $3 d_{x}{ }^{2}-y^{2}$ and $3 d_{z}{ }^{2}-r^{2}$ states, pushing the $3 d_{z^{2}}-r^{2}$ states closer to the $E_{f}$ leading to an increasing number of holes on $3 \mathrm{~d}_{\mathrm{z}}{ }^{2}-\mathrm{r}^{2}$ orbitals.

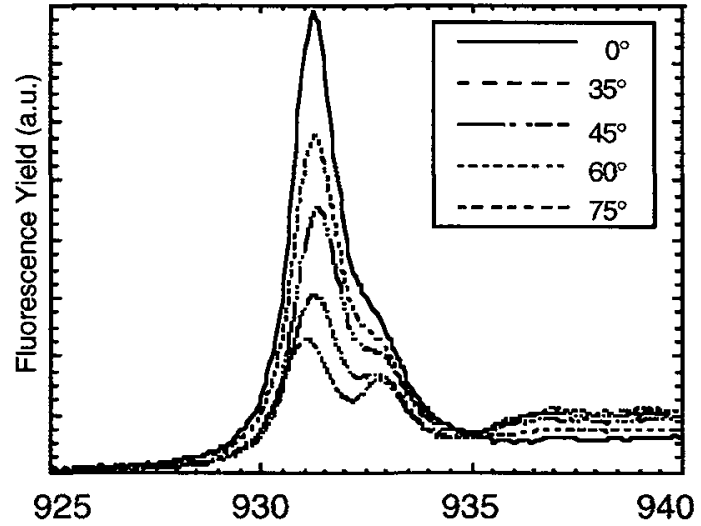

Fig.1: Polarized FY $\mathrm{Cu} \mathrm{L}_{3}$-edges of a $\mathrm{Tl}(2212)$ thin film synthesized under high oxygen pressure (70 bars).

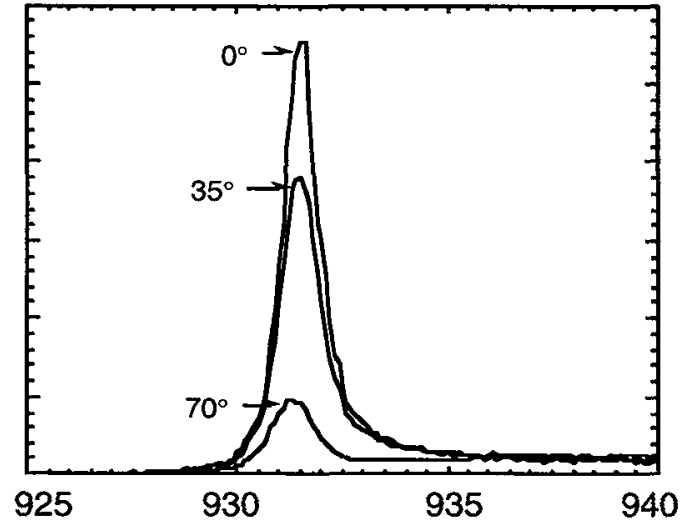

Fig. 2: Polarized FY $\mathrm{Cu} \mathrm{L}_{3}$-edges of a T1(2212) thin film synthesized under low oxygen pressure (1 bar).

The slight decrease in Tc observed in the overdoped sample is related to the variation in the in-plane density of holes crossing the optimum value [5,6] and is not due to the observed increase in amount of holes having $\mathrm{Cu} 3 \mathrm{~d}_{\mathrm{z}}{ }^{2}-\mathrm{r}{ }^{2}$ symmetry. This clearly implies that these states are neither beneficial nor detrimental to the cause of superconductivity as concluded by Pellegrin et al [7] for bi-layer and three layer Tl-cuprates.

\subsubsection{Role of out-of-plane copper orbitals}

The Cu L3-edge spectra of $\mathrm{Tl}$ (2212) thin films have been recorded at room temperature using bulk sensitive fluorescence yield (FY) mode. The spectra obtained using the FY mode were corrected following the work of Tröger et al [8] which describes a full correction method of the self-absorption in soft flurorescence extended $x$-ray fine structure. The correction enhances the white line $13 d^{9}>$ intensity with respect to the $13 d^{9} \underline{L}>$ one and decreases the $13 d^{9}>$ line width with respect to the total yield spectrum. The spectra of the two $\mathrm{Tl}(2212)$ thin films synthesized in vacuum and under medium oxygen pressure at different orientations obtained after the correction are shown in figures 2 and 3 respectively. In order to obtain covalent and doping hole

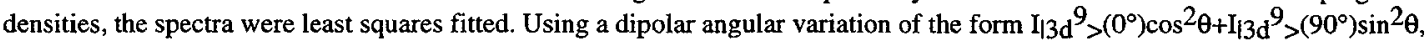
the intensity can be extrapolated to $\theta=90^{\circ}$. For the vacuum synthesized thin film, the density of covalent holes along the $z$-axis was estimated to be $\approx 10 \%$. However, no $13 \mathrm{~d}^{9} \underline{\mathrm{L}}>$ doping holes were found to have $\mathrm{z}$-symmetry. On the other hand the thin film synthesised in medium pressure of oxygen ( 10 bars) gives $12 \%$ and $\approx 2 \%$ covalent and doping hole densities along the $z$-axis respectively. The total $\left(3 \mathrm{~d}^{9}+3 \mathrm{~d}^{9} \mathrm{~L}\right)$ density of holes in out-of-plane $\mathrm{Cu}$ orbitals in the case of $\mathrm{Tl}(2212)$ thin film synthesized under medium pressure of oxygen turns out to be $14 \%$, which is $5 \%$ more with respect to the vacuum synthesized Tl(2212) thin film. The $T_{c}$ decreases from $105 \mathrm{~K}$ to $100 \mathrm{~K}$, which as we have conclusively proved in our previous work [5,9] is due to the change in density of in-plane doping holes which changes from 0.15 to 0.20 in the two films synthesised in vacuum and medium oxygen pressure. As can be seen, the value of $n_{h}$ in both $T l(2212)$ thin films is more than the optimum value $(\approx 0.12)$ obtained in our previous study $[9,10]$ which resulted in the value of Tc which is less than the highest observed in this system. On overdoping, the asymmetry of crystal field is reduced by the change in local environment of $\mathrm{Cu}$. Previous works have shown as a general trend that the $c$ unit cell parameter in thallium bilayer cuprates decrease under doping and vice-versa indicating a consequent decrease of the $\mathrm{Cu}-\mathrm{O}$ apical distance [10]. This reduces the splitting between $3 \mathrm{~d}_{\mathrm{x}}^{2}-\mathrm{y}^{2}$ and $3 \mathrm{~d}_{z}{ }^{2}-\mathrm{r}^{2}$ states,

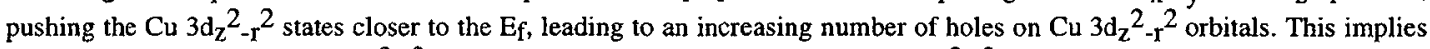
that at high doping a band of $3 d_{z}{ }^{2}-r^{2}$ character starts emerging at the $E_{f}$. These $3 d_{z^{2}}-r^{2}$ orbital mixes with $O 2 p$ valence band which is indicated by the presence of doping hole density along the $\mathrm{z}$-axis in the $\mathrm{Tl}(2212)$ thin film synthesized under medium pressure of oxygen, corresponding to an expected $13 d^{9} z^{2}-r^{2} \underline{L}>$ configuration. Hence, after a certain amount of doping, exchange do take place between $\mathrm{Cu}$ and its apical oxygens and as suggested by our recent $\mathrm{OK}$ and $\mathrm{T} 1 \mathrm{~L}_{3}$-edge measurements [11], higher amount of doping holes could be stabilized in the Tl-O layers via these apical oxygens.

However, the presence of high density of holes having $\mathrm{Cu} 3 \mathrm{~d}_{\mathrm{z}}{ }^{2}-\mathrm{r}^{2}$ character does not seem to play an active role in the mechanism of superconductivity as the small decrease in $\mathrm{Tc}$ can be explained in terms of the variation in density of doping holes having in-plane symmetry. These results are in agreement with the study of Pellegrin et al [7], which leads to the conclusion that no direct correlation exists between $\mathrm{Cu} 3 \mathrm{~d}_{\mathrm{z}}{ }^{2}-\mathrm{r}^{2}$ and $\mathrm{T}_{\mathrm{c}}$ in $\mathrm{T}$ bi-layer and three layer superconductors though their study was restricted to only one doping hole concentration. 


\subsection{O K- AND T1 L3-EDGES}

Figure 4 shows polarized OK-edge spectra using bulk sensitive FY mode at different orientations on the same thin film synthesized under high oxygen pressure as in figure 1 . The $E / /(a, b)$ and $E / / c$ measurements probe unoccupied states with $O$ $2 \mathrm{p}_{\mathrm{x}, \mathrm{y}}$ and $\mathrm{O} 2 \mathrm{p}_{\mathrm{z}}$ characters respectively.

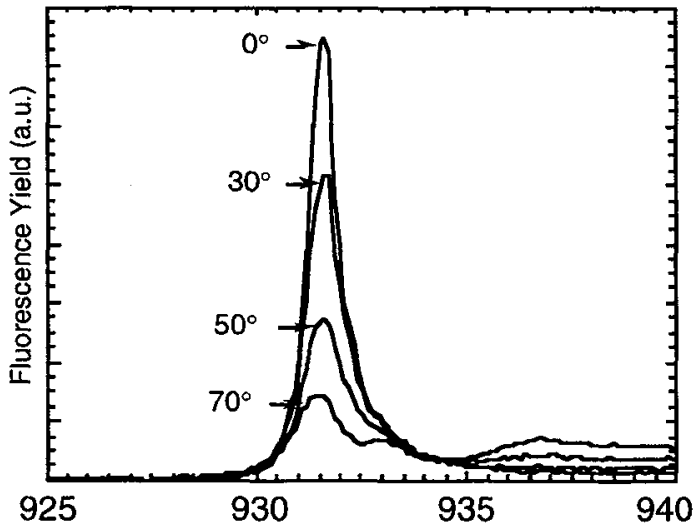

Fig.3: Polarized FY $\mathrm{Cu} \mathrm{L}_{3}$-edges of a $\mathrm{Tl}(2212)$ thin film synthesized under medium oxygen pressure (10 bars).

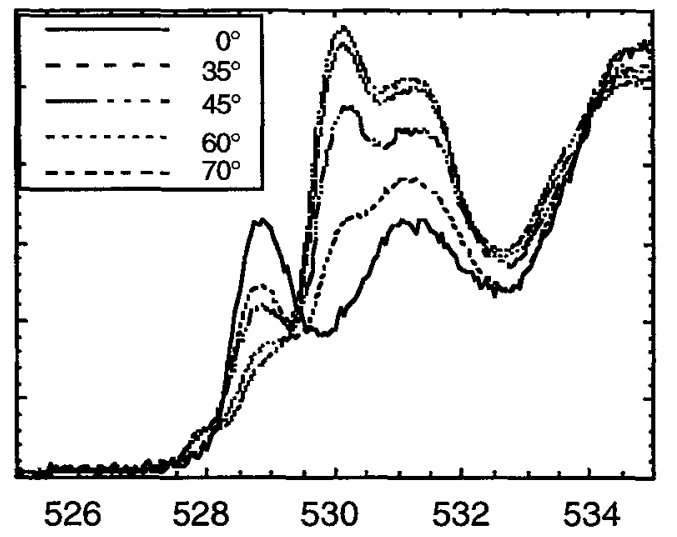

Fig.4: Polarized FY O K-edges of a Tl(2212) thin film synthesized under high oxygen pressure (70 bars).

The E//a,b spectrum consists of two features, a prepeak at 528.4 and a broad feature around $530.7 \mathrm{eV}$. The prepeak in E//(a,b) spectrum is attributed to transitions into the unoccupied in-plane $O$ (1) $2 p_{x, y}$ states. These empty states represent the oxygen contribution to the doping hole densities, as described through the $13 \mathrm{~d}^{9} \underline{\mathrm{L}}>$ representation and referred to ligand band in the effective one-band Hubbard model [10] extended to O K-edge. We have estimated its intensity for the $90^{\circ}(\mathrm{E} / / \mathrm{c})$ incidence spectrum and found it to be negligible within the experimental error. This confirms that the doping holes have predominantly $\mathrm{O} 2 \mathrm{px}, \mathrm{y}$ symmetry.

Changing the polarization from $E / /(a, b)$ to $E / / c$, the intensity of the prepeak at 528.4 decreases drastically and another peak and a broad structure appear around 529.7 and $530.8 \mathrm{eV}$ respectively. Taking into consideration the chemical shifts calculated by band structure calculations $[4,12]$ for Tl-cuprates, the features observed at 529.7 and $530.8 \mathrm{eV}$ in the spectrum recorded at $80^{\circ}$ incidence are ascribed to $O(2)$ and $O(3) 2 \mathrm{p}$ states respectively. According to the LDA calculations, the essential contribution to the spectral weight for $O(2)$ and $O(3)$ atoms comes from the states forming narrow peaks in the $2 p$ density of states (DOS). These DOS at the Fermi level consist of $\mathrm{O}(2)-\mathrm{Tl}-\mathrm{O}(3)$ band formed by the hybridization of $\mathrm{Tl} 6 \mathrm{~s}$ and $\mathrm{Tl} 5 \mathrm{~d} 3 \mathrm{z}^{2}-\mathrm{r}^{2}$ orbitals and $O(2)$ and $O(3) 2 p_{z}$ orbitals and therefore have predominantly $O 2 p_{z}$ character. The hybridization is quite strong due to relatively short distances between $\mathrm{Tl}$ and apical $\mathrm{O}$ atoms $(\mathrm{T}-\mathrm{O}(2)=2.05 \AA$ and $\mathrm{Tl}-\mathrm{O}(3)=2.42 \AA$ ). Here we would like to point out that the intensity of the feature at $530.8 \mathrm{eV}$, corresponding to the $\mathrm{Tl}-\mathrm{O}(3)$ bond, is high, specially on the spectrum recorded at $80^{\circ}$ incidence (Figure 8 ), as compared to the ones reported on under or optimally doped $\mathrm{Tl}(2212)$ thin films and single crystals [7]. This suggests that extra oxygens could be stabilized in the T]-O layers in these synthesis conditions under high oxygen pressure.

The origin of the broad structure at $530.7 \mathrm{eV}$ in $\mathrm{E} / / \mathrm{a}, \mathrm{b}$ spectrum is still not clear. As suggested by Pellegrin et al [7] it may be composed of states with $\mathrm{O} 2 \mathrm{px}, \mathrm{y}$ character originating from the Upper Hubbard Band (UHB) related to $\mathrm{CuO}_{2}$ planes and $O(2)$ and/or $O(3)$ atoms. From our spectrum it appears that contribution from $O(3)$ states is more than the $O(2)$ states. Also, polarized XAS experiments at T1 L3-edge on the same thin films have shown that, due to the short Tl-O(2) distances, a strong charge tranfer takes place from the $O(2)$ apical oxygen and, to a smaller extent, from the $O(3)$ oxygen to the $T 1$ along the c-axis. This is in agreement to our earlier studies [1] and also with band structure calculations for this system [11] which predict that the electron pockets at $E_{f}$ are formed from an antibonding band composed of $O(2) 2 p_{z}$ and $O(3) 2 p_{z}$ orbitals

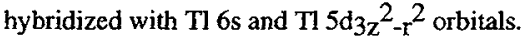

\section{References}

[1] F.Studer, N.Merrien, C.Martin, C.Michel, B.Raveau and A.Fontaine, Physica C 178 (1991) 324.

[2] R.M.Hazen, Physical Properties of High Temperature Superconductors II, Ed. D.M.Ginsberg (World Scientific, 1990$) 121$.

[3] H.Romberg, N.Nücker, M.Alexander, J.Fink, D.Hahn, T.Zetterer, H.H.Otto and K.F.Renk, Phys.Rev B41 (1990) 2609.

[4] J.Yu, S.Massida and A.J.Freeman, Physica C152 (1988) 273.

[5] N.Merrien, L.Coudrier, C.Martin, A.Maignan, F.Studer and A-M.Flank, Phys.Rev.B49(14) (1994) 9906.

[6] C.Gasser, A.Taffin, B.Mercey, F.Studer, H.Murray and P.Berger, J.Sol.Stat.Chem.(1996) in press.

[7] E.Pellegrin et al., Phys.Rev.B48 (1993) 10520

[8] L.Tröger et al., Phys.Rev.B46 (1992) 3283.

[9] C.Gasser, F.Studer, A.Maignan, H.Murray and M.Pompa, J.Sol.Stat.Chem.(1996) in press.

[10] A.Maignan et al., Physica C 170 (1990) 350.

[11] C.Gasser, PhD Thesis, University of Caen (1995).

[12] P.Marksteiner et al., Phys.Rev.B39 (1989) 2894. 